\title{
On the Fock Kernel for the Generalized Fock Space and Generalized Hypergeometric Series
}

\author{
Jong-Do Park \\ Department of Mathematics, Kyung Hee University, Seoul 02447, Republic of Korea \\ Correspondence should be addressed to Jong-Do Park; mathjdpark@khu.ac.kr
}

Received 27 April 2021; Accepted 7 August 2021; Published 23 August 2021

Academic Editor: Guozhen Lu

Copyright (c) 2021 Jong-Do Park. This is an open access article distributed under the Creative Commons Attribution License, which permits unrestricted use, distribution, and reproduction in any medium, provided the original work is properly cited.

In this paper, we compute the reproducing kernel $B_{m, \alpha}(z, w)$ for the generalized Fock space $F_{m, \alpha}^{2}(\mathbb{C})$. The usual Fock space is the case when $m=2$. We express the reproducing kernel in terms of a suitable hypergeometric series ${ }_{1} F_{q}$. In particular, we show that there is a close connection between $B_{4, \alpha}(z, w)$ and the error function. We also obtain the closed forms of $B_{m, \alpha}(z, w)$ when $m=$ $1,2 / 3,1 / 2$. Finally, we also prove that $B_{m, \alpha}(z, z) \sim e^{\alpha|z|^{m}}|z|^{m-2}$ as $|z| \longrightarrow \infty$.

\section{Introduction}

For any fixed parameter $\alpha>0$, we consider

$$
d \lambda_{m}(z):=d \lambda_{m, \alpha}(z)=c_{m, \alpha} e^{-\alpha|z|^{m}} d A(z)
$$

where $d A(z)$ is the Euclidean area measure on the complex plane $\mathbb{C}$. Here, $c_{m, \alpha}$ is a normalizing constant so that $d \lambda_{m, \alpha}$ is a probability measure on $\mathbb{C}$.

We call the generalized Fock space $F_{m}^{2}(\mathbb{C}):=F_{m, \alpha}^{2}(\mathbb{C})$ the set of all entire functions $f$ in $L^{2}\left(\mathbb{C}, d \lambda_{m}(z)\right)$. It is easy to see that $F_{m}^{2}(\mathbb{C})$ is a Hilbert space with the inner product:

$$
\left.\langle f, g\rangle:=\int_{C} f(z) g \overline{(z}\right) d \lambda_{m}(z)
$$

Let $\left\{\phi_{j}(\cdot): j \in \mathbb{N}\right\}$ be the countable orthonormal basis for $F_{m}^{2}(\mathbb{C})$. Then, the generalized Fock kernel $B_{m}(z, w):=B_{m, \alpha}($ $z, w)$ for $F_{m}^{2}(\mathbb{C})$ is defined by

$$
\left.B_{m}(z, w):=\sum_{j \in \mathbb{N}} \phi_{j}(z) \phi_{j} \overline{(} w\right) .
$$

If $m=2$, then $F_{2}^{2}(\mathbb{C})$ is the usual Fock space. In fact, it is well known that $B_{2}(z, w)=e^{\alpha z \bar{w}}$ for $z, w \in \mathbb{C}$. See the detailed properties on the usual Fock space in the book [1] written by Zhu. In fact, the explicit form of $B_{2}(z, w)$ is very useful for studying the properties of the Fock space in [2].

In this paper, we focus on the following natural question.

Question: compute the Fock kernel $B_{m}(z, w)$ for any positive rational number $m$.

In the theory of the Bergman kernel, it is difficult to find the closed form of the Bergman kernel for a general domain. Instead, in the case of a complex ellipsoid or similar domains, one can see the expression of the Bergman kernel in terms of the hypergeometric series in $[3,4]$.

The generalized hypergeometric series ${ }_{p} F_{q}\left(a_{1}, \cdots, a_{p} ; b_{1}\right.$ $\left., \cdots, b_{q} ; x\right)$ is defined by

$$
{ }_{p} F_{q}\left(a_{1}, \cdots, a_{p} ; b_{1}, \cdots, b_{q} ; x\right)=\sum_{k=0}^{\infty} \frac{\left(a_{1}\right)_{k} \cdots\left(a_{p}\right)_{k}}{\left(b_{1}\right)_{k} \cdots\left(b_{q}\right)_{k}} \frac{x^{k}}{k !},
$$

where $(a)_{k}$ is the Pochhammer symbol defined by

$$
(a)_{k}=\left(\begin{array}{ll}
\frac{\Gamma(a+k)}{\Gamma(a)}=a(a+1) \cdots(a+k-1), & k \geq 1, \\
1, & k=0 .
\end{array}\right.
$$

If $p=q+1$, then the series converges for $|x|<1$ and 
diverges for $|x|>1$. If $p<q+1$, then the series converges for all $x$. If $p>q+1$, then the series converges only at $x=0$.

It is well known that the Bergman kernel for the complex ellipsoid

$$
D\left(p_{1}, \cdots, p_{n}\right):=\left\{\left(z_{1}, \cdots, z_{n}\right) \in \mathbb{C}^{n}:\left|z_{1}\right|^{2 p_{1}}+\cdots+\left|z_{n}\right|^{2 p_{n}}<1\right\}
$$

is closely connected with ${ }_{2} F_{1}$ and its higher dimensional hypergeometric series (Appell hypergeometric series or Lauricella hypergeometric series). Using the theory of the hypergeometric series, new formulas of the Bergman kernel have been computed in $[3,5-7]$.

Recently, new interesting generalized Fock spaces have been studied. In [8], Gonessa investigated the duality on the generalized Fock space with respect to the minimal norm. In [9], one can see the boundedness of the Bergman projection on the generalized Fock-Sobolev space with respect to $d \lambda_{m}(z)$. But they did not obtain the explicit forms of the integral kernel. In [10], Cho et al. computed the Fock kernel for the space with respect to $d \mu_{\alpha}(z)=c_{\alpha}|z|^{2 \alpha} e^{-|z|^{2}} d V$ $(z)$. For $\alpha>0$, the kernel $K_{\alpha}(z, w)$ is represented by $K_{\alpha}(z$, $w)={ }_{1} F_{1}(n, n+\alpha ;\langle z, w\rangle)$ for $z, w \in \mathbb{C}^{n}$.

The main theorem of this paper is the following. At first, we consider the case when $m$ is a positive integer.

Theorem 1. Let $m$ be any positive integer and let $\zeta:=\alpha^{2 / m} z \bar{w}$.

(i) If $m$ is even, then

$$
B_{m}(z, w)=\frac{m \alpha^{2 / m}}{2 \pi} \sum_{r=0}^{(m / 2)-1} \frac{\zeta^{r}}{\Gamma((2 r+2) / m)}{ }_{1} F_{1}\left(1 ; \frac{2 r+2}{m} ; \zeta^{m / 2}\right) .
$$

(ii) If $m$ is odd, then

$$
B_{m}(z, w)=\frac{m \alpha^{2 / m}}{2 \pi} \sum_{r=0}^{m-1} \frac{\zeta^{r}}{\Gamma((2 r+2) / m)}{ }_{1} F_{2}\left(1 ; \frac{r+1}{m}, \frac{r+1}{m}+\frac{1}{2} ; \frac{\zeta^{m}}{4}\right) .
$$

Now, we generalize to the case when $m$ is a positive rational number.

Theorem 2. Let $m$ be any positive rational number and let $\zeta:=\alpha^{2 / m} z \bar{w}$.

(i) If $m=2 p / q$, where $2 p$ and $q$ are relatively prime, then

$$
B_{m}(z, w)=\frac{m \alpha^{2 / m}}{2 \pi} \sum_{r=0}^{p-1} \frac{\zeta^{r}}{\Gamma(q(r+1) / p)}{ }_{1} F_{q}\left(1 ; \frac{\mathbf{r}+1}{\mathbf{p}}+\frac{\overrightarrow{\mathbf{j}}}{\mathbf{q}} ; \frac{\zeta^{p}}{q^{q}}\right),
$$

where

$$
\frac{\mathbf{r}+1}{\mathbf{p}}+\frac{\overrightarrow{\mathbf{j}}}{\mathbf{q}}=\left(\frac{r+1}{p}, \frac{r+1}{p}+\frac{1}{q}, \frac{r+1}{p}+\frac{2}{q}, \cdots, \frac{r+1}{p}+\frac{q-1}{q}\right) .
$$

(ii) If $m=(2 p+1) / q$, where $2 p+1$ and $q$ are relatively prime, then

$$
\begin{aligned}
B_{m}(z, w)= & \frac{m \alpha^{2 / m}}{2 \pi} \sum_{r=0}^{2 p} \frac{\zeta^{r}}{\Gamma(2 q(r+1) /(2 p+1))}{ }_{1} F_{2 q} \\
& \cdot\left(1 ; \frac{\mathbf{r}+1}{2 \mathbf{p}+1}+\frac{\overrightarrow{\mathbf{j}}}{2 \mathbf{q}} ; \frac{\zeta^{2 p+1}}{(2 q)^{2 q}}\right),
\end{aligned}
$$

where

$$
\frac{\mathbf{r}+1}{2 \mathbf{p}+1}+\frac{\overrightarrow{\mathbf{j}}}{2 \mathbf{q}}=\left(\frac{r+1}{2 p+1}, \frac{r+1}{2 p+1}+\frac{1}{2 q}, \cdots, \frac{r+1}{2 p+1}+\frac{2 q-1}{2 q}\right) .
$$

In particular, if $m=4$, then there is a close connection between $B_{4}(z, w)$ and the error function.

Theorem 3. Let $\alpha>0$. Then,

$$
B_{4}(z, w)=\frac{2 \alpha}{\pi} z \bar{w} e^{\alpha(z \bar{w})^{2}}(\operatorname{erf}(\sqrt{\alpha} z \bar{w})+1)+\frac{2 \sqrt{\alpha}}{\pi \sqrt{\pi}},
$$

where erf $(x)$ is the error function denoted by

$$
\operatorname{erf}(x)=\frac{2}{\sqrt{\pi}} \int_{0}^{x} e^{-t^{2}} d t
$$

In general, it is difficult to find the closed form of the generalized hypergeometric series ${ }_{p} F_{q}$. Using the hypergeometric series in Theorems 1 and 2, we obtain the following closed forms for $m=1,2 / 3,1 / 2$.

Theorem 4. Let $\alpha>0$. Then,

(i) $B_{1}(z, w)=(\alpha / 2 \pi)\left(\sinh \left(\alpha(z \bar{w})^{1 / 2}\right) /(z \bar{w})^{1 / 2}\right)$,

(ii) $B_{2 / 3}(z, w)=\alpha e^{-\alpha / 2(z \bar{w})^{1 / 3}} / 9 \pi(z \bar{w})^{2 / 3}\left\{e^{3 / 2 \alpha(z \bar{w})^{1 / 3}}-2\right.$ $\left.\sin \left((\sqrt{3} / 2) \alpha(z \bar{w})^{1 / 3}+(\pi / 6)\right)\right\}$,

(iii) $B_{1 / 2}(z, w)=\alpha / 8 \pi(z \bar{w})^{3 / 4}\left\{\sinh \left(\alpha(z \bar{w})^{1 / 4}\right)-\sin (\alpha\right.$ $\left.\left.(z \bar{w})^{1 / 4}\right)\right\}$.

Finally, we discuss the asymptotic behavior of the Fock kernel. Now, we write $A(x) \sim B(x)$ if $A(x) / B(x)$ converges to nonzero constant as $x$ goes to some number or infinity. Denote $K_{D}(z, w)$ by the Bergman kernel for the bounded 
domain $D \subset \mathbb{C}^{n}$. It is a well-known fact that $K_{D}(z, z)$ diverges to infinity under some condition. More precisely, if $d(z)$ is the distance to the boundary $b D$, then

$$
K_{D}(z, z) \sim d(z)^{n+1}
$$

as $z$ approaches the strongly pseudoconvex boundary point $p \in b D$.

Using the properties of the incomplete gamma function, we can obtain the similar result also for the generalized Fock space.

Theorem 5. Let $m$ be any positive even integer. Then,

$$
B_{m}(z, z) \sim e^{\alpha|z|^{m}}|z|^{m-2} a s|z| \longrightarrow \infty .
$$

Remark 6. The usual Fock kernel $B_{2}(z, w)=e^{\alpha z \bar{w}}$ is very simple but plays an important role in the research of the function theoretic properties of the Fock space $F_{2, \alpha}^{2}(\mathbb{C})$. Theorems 1 and 2 in this paper are the first result on the generalized Fock space $F_{m, \alpha}^{2}(\mathbb{C})$ for any $m \neq 2$. Also, we hope that the explicit formulas in Theorems 3 and 4 can give a clue on studying optimal pointwise estimates for $B_{m}(z, w)$ for some $m$.

\section{Computation of $B_{m}(z, w)$}

Consider $d \lambda_{m}(z)=c_{m, \alpha} e^{-\alpha|z|^{m}} d A(z)$, where $c_{m, \alpha}$ is a normalizing constant so that $d \lambda_{m}(z)$ is a probability measure on $\mathbb{C}$. In fact, we can obtain $c_{m, \alpha}$ from the following lemma.

Lemma 7. For any nonnegative integers $k$, we have

$$
\left\|z^{k}\right\|^{2}=\frac{2 \pi}{m \alpha^{(2 k+2) / m}} \Gamma\left(\frac{2 k+2}{m}\right)
$$

where $\Gamma(\cdot)$ is the usual gamma function. In particular, we have

$$
c_{m, \alpha}=\frac{m \alpha^{2 / m}}{2 \pi \Gamma(2 / m)} .
$$

Proof. Recall that the usual gamma function $\Gamma$ is defined by

$$
\Gamma(z)=\int_{0}^{\infty} x^{z-1} e^{-x} d x, \mathfrak{R}(z)>0 .
$$

Using the polar coordinate change, we have

$$
\left\|z^{k}\right\|^{2}=\int_{\mathbb{C}}\left|z^{k}\right|^{2} e^{-\alpha|z|^{m}} d A(z)=2 \pi \int_{0}^{\infty} r^{2 k+1} e^{-\alpha r^{m}} d r .
$$

If we can substitute $s=\alpha r^{m}$, then by (19)

$$
\begin{aligned}
\left\|z^{k}\right\|^{2} & =2 \pi \int_{0}^{\infty}\left(\frac{s}{\alpha}\right)^{(2 k+1) / m} e^{-s} \frac{1}{m \alpha^{1 / m}} s^{(1 / m)-1} d s \\
& =\frac{2 \pi}{m \alpha^{(2 k+2) / m}} \int_{0}^{\infty} s^{(2 k+2) / m-1} e^{-s} d s=\frac{2 \pi}{m \alpha^{(2 k+2) / m}} \Gamma\left(\frac{2 k+2}{m}\right) .
\end{aligned}
$$

It completes the proof.

It follows that the reproducing kernel $B_{m}(z, w)$ is written as

$$
B_{m}(z, w)=\sum_{k=0}^{\infty} \frac{(z \bar{w})^{k}}{\left\|z^{k}\right\|^{2}}=\frac{m \alpha^{2 / m}}{2 \pi} \sum_{k=0}^{\infty} \frac{\left(\alpha^{2 / m} z \bar{w}\right)^{k}}{\Gamma((2 k+2) / m)}
$$

Throughout this paper, we are focusing on computing the function

$$
G_{m}(\zeta):=\sum_{k=0}^{\infty} \frac{\zeta^{k}}{\Gamma((2 k+2) / m)}
$$

Then, we have

$$
B_{m}(z, w)=\frac{m \alpha^{2 / m}}{2 \pi} G_{m}\left(\alpha^{2 / m} z \bar{w}\right)
$$

Remark 8. If $m=2$, then $G_{2}(\zeta)=\sum_{k=0}^{\infty} \zeta^{k} / k !=e^{\zeta}$. In this case,

$$
B_{2}(z, w)=\frac{\alpha}{\pi} e^{\alpha z \bar{w}}
$$

which is just the usual Fock kernel.

Now, we investigate the relation between $G_{m}(\zeta)$ and generalized hypergeometric series for any positive rational number $m$.

\section{Proof of Theorem 1}

In this section, we express the Fock kernel $B_{m}(z, w)$ in terms of the suitable hypergeometric series ${ }_{p} F_{q}$ when $m$ is a positive integer. The crucial term for computing the form of $B_{m}(z, w)$ is $\Gamma((2 k+2) / m)$.

3.1. Proof of Theorem 1 (i). Assume that $m$ is an even integer. Let $m=2 p$ for some $p \in \mathbb{N}$. Then, we have

$$
G_{m}(\zeta)=\sum_{k=0}^{\infty} \frac{\zeta^{k}}{\Gamma((k+1) / p)}
$$

Theorem 1 (i) can be easily proven by the following proposition using (24). 
Proposition 9. Let $m$ be any even positive integer, and let $\zeta$ $:=\alpha^{2 / m} z \bar{w}$. Then, we have

$$
G_{m}(\zeta)=\sum_{r=0}^{(m / 2)-1} \frac{\zeta^{r}}{\Gamma((2 r+2) / m)} \Phi\left(1 ; \frac{2 r+2}{m} ; \zeta^{m / 2}\right)
$$

where $\Phi(a ; b ; x):={ }_{1} F_{1}(a ; b ; x)$ is the confluent hypergeometric series.

Proof. Note that there exist unique integers $\ell$ and $r$ such that $k=p \ell+r$ with $0 \leq r \leq p-1$. Thus, we have

$$
G_{m}(\zeta)=\sum_{r=0}^{p-1} \zeta^{r} \sum_{\ell=0}^{\infty} \frac{\left(\zeta^{p}\right)^{\ell}}{\Gamma(\ell+((r+1) / p))}=\sum_{r=0}^{\infty} \frac{\zeta^{r}}{\Gamma((r+1) / p)} \sum_{\ell=0}^{\infty} \frac{\left(\zeta^{p}\right)^{\ell}}{((r+1) / p)_{\ell}} .
$$

Note that

$$
\Phi(1 ; b ; x)={ }_{1} F_{1}(1 ; b ; x)=\sum_{k=0}^{\infty} \frac{1}{(b)_{\ell}} x^{\ell}
$$

It follows that

$$
G_{m}(\zeta)=\sum_{r=0}^{p-1} \frac{\zeta^{r}}{\Gamma((r+1) / p)} \Phi\left(1 ; \frac{r+1}{p} ; \zeta^{p}\right)
$$

which completes the proof.

3.2. Proof of Theorem 1 (ii). Assume that $m$ is an odd integer. Let $m=2 p+1$ for some $p \in \mathbb{N}$. Then,

$$
G_{m}(\zeta)=\sum_{k=0}^{\infty} \frac{\zeta^{k}}{\Gamma((2 k+2) /(2 p+1))}
$$

Theorem 1 (ii) can be easily proven by the following proposition using (24).

Proposition 10. Let $m$ be any odd positive integer, and let $\zeta:=\alpha^{2 / m} z \bar{w}$. Then,

$$
G_{m}(\zeta)=\sum_{r=0}^{m-1} \frac{\zeta^{r}}{\Gamma((2 r+2) / m)}{ }_{1} F_{2}\left(1 ; \frac{r+1}{m}, \frac{r+1}{m}+\frac{1}{2} ; \frac{\zeta^{m}}{4}\right) .
$$

Proof. Note that there exist unique integers $\ell$ and $r$ such that $k=(2 p+1) \ell+r$ with $0 \leq r \leq 2 p$. Then,

$$
G_{m}(\zeta)=\sum_{r=0}^{2 p} \zeta^{r} \sum_{\ell=0}^{\infty} \frac{\left(\zeta^{2 p+1}\right)^{\ell}}{\Gamma(2 \ell+((2 r+2) /(2 p+1)))} .
$$

Now, we will use the identity

$$
\Gamma(2 \ell+2 t)=2^{2 \ell}(t)_{\ell}\left(t+\frac{1}{2}\right)_{\ell} \Gamma(2 t)
$$

for any nonnegative integer $\ell$ and $t \in \mathbb{R}$. In fact, the identity (34) can be proven by

$$
\begin{aligned}
\frac{\Gamma(2 \ell+2 t)}{\Gamma(2 t)} & =(2 t)(2 t+1) \cdots(2 t+2 \ell-1) \\
& =2^{2 \ell} t(t+1) \cdot(t+\ell-1)\left(t+\frac{1}{2}\right)\left(t+\frac{3}{2}\right) \cdots\left(t+\frac{2 \ell-1}{2}\right) \\
& =2^{2 \ell}(t)_{\ell}\left(t+\frac{1}{2}\right)_{\ell} .
\end{aligned}
$$

Then, by (34), we have

$$
\begin{aligned}
G_{m}(\zeta) & =\sum_{r=0}^{2 p} \frac{\zeta^{r}}{\Gamma((2 r+2) /(2 p+1))} \sum_{\ell=0}^{\infty} \frac{1}{((r+1) /(2 p+1))_{\ell}(((r+1) / 2 p+1)+1 / 2)_{\ell}}\left(\frac{\zeta^{2 p+1}}{4}\right)^{\ell} \\
& =\sum_{r=0}^{2 p} \frac{\zeta^{r}}{\Gamma((2 r+2) /(2 p+1))}{ }_{1} F_{2}\left(1 ; \frac{r+1}{2 p+1}, \frac{r+1}{2 p+1}+\frac{1}{2} ; \frac{\zeta^{2 p+1}}{4}\right),
\end{aligned}
$$

since ${ }_{1} F_{2}\left(1 ; b_{1}, b_{2} ; x\right)=\sum_{\ell=0}^{\infty} x^{\ell} /\left(b_{1}\right)_{\ell}\left(b_{2}\right)_{\ell}$.

\section{Proof of Theorem 2}

In this section, we focus on computing $G_{m}$ when $m$ is a positive rational number.

4.1. Proof of Theorem 2 (i): Even Numerator. Let $m=2 p / q$, where $2 p$ and $q$ are relatively prime. Then, we have

$$
G_{m}(\zeta)=\sum_{k=0}^{\infty} \frac{\zeta^{k}}{\Gamma((k+1) q / p)}=\sum_{r=0}^{p-1} \zeta^{r} \sum_{\ell=0}^{\infty} \frac{\left(\zeta^{p}\right)^{\ell}}{\Gamma(q \ell+(q(r+1) / p))},
$$

where $k=p \ell+r$ with $0 \leq r \leq p-1$.

Lemma 11. The gamma function $\Gamma$ satisfies the identity

$\Gamma(x) \Gamma\left(x+\frac{1}{n}\right) \Gamma\left(x+\frac{2}{n}\right) \cdots \Gamma\left(x+\frac{n-1}{n}\right)=(2 \pi)^{n-1 / 2} n^{(1 / 2)-n x} \Gamma(n x)$.

Using the above lemma, we can prove the following.

\section{Lemma 12.}

$$
\Gamma(q \ell+q t)=q^{q \ell} \prod_{j=0}^{q-1}\left(t+\frac{j}{q}\right)_{\ell} \Gamma(q t) .
$$

Proof. We will prove it in two different methods. Using the property $\Gamma(x+1)=x \Gamma(x)$, we have

$$
\frac{\Gamma(q \ell+q t)}{\Gamma(q t)}=\prod_{i=0}^{q \ell-1}(q t+i)=q^{q \ell} \prod_{i=0}^{q \ell-1}\left(t+\frac{i}{q}\right) .
$$

Then, there exists $x, y \in \mathbb{Z}$ such that $i=q j+y$ with $0 \leq j$ 
$\leq \ell-1$ and $0 \leq y \leq q-1$. It follows that

$$
\prod_{i=0}^{q \ell-1}\left(t+\frac{i}{q}\right)=\prod_{j=0}^{q-1} \prod_{x=0}^{\ell-1}\left(t+\frac{j}{q}+x\right)=\prod_{j=0}^{q-1}\left(t+\frac{j}{q}\right)_{\ell} .
$$

It can be proven also using Lemma 11. Note that

$$
\begin{aligned}
\Gamma(q \ell+q t) & =\frac{\Gamma(\ell+t) \Gamma(\ell+t+(1 / q)) \cdots \Gamma(\ell+t+((q-1) / q))}{(2 \pi)^{(q-1) / 2} q^{(1 / 2)-q(\ell+t)}} \\
& =\frac{\Gamma(t) \Gamma(t+1 / q) \cdots \Gamma \Gamma(t+(q-1) / q)}{(2 \pi)^{(q-1) / 2} q^{(1 / 2)-q(\ell+t)}}(t)_{e}\left(t+\frac{1}{q}\right)_{e} \cdots\left(t+\frac{q-1}{q}\right)_{e} \\
& =\frac{(2 \pi)^{(q-1) / 2} q^{(1 / 2)-q t} \Gamma(q t)}{(2 \pi)^{(q-1) / 2} q^{(1 / 2)-q(\ell+t)}}(t)_{e}\left(t+\frac{1}{q}\right)_{e} \cdots\left(t+\frac{q-1}{q}\right)_{e} \\
& =q^{q \ell} \Gamma(q t)(t)_{e}\left(t+\frac{1}{q}\right)_{e} \cdots\left(t+\frac{q-1}{q}\right)_{e} .
\end{aligned}
$$

Now, we prove Theorem 2 (i) using Lemma 12.

Theorem 13 (Theorem 2 (i) again). Let $m=2 p / q$, where $2 p$ and $q$ are relatively prime. Then,

$$
G_{m}(\zeta)=\sum_{r=0}^{p-1} \frac{\zeta^{r}}{\Gamma(q(r+1) / p)}{ }_{1} F_{q}\left(1 ; \frac{\mathbf{r}+1}{\mathbf{p}}+\frac{\overrightarrow{\mathbf{j}}}{\mathbf{q}} ; \frac{\zeta^{p}}{q^{q}}\right)
$$

where

$$
\frac{\mathbf{r}+1}{\mathbf{p}}+\frac{\overrightarrow{\mathbf{j}}}{\mathbf{q}}=\left(\frac{r+1}{p}, \frac{r+1}{p}+\frac{1}{q}, \frac{r+1}{p}+\frac{2}{q}, \cdots, \frac{r+1}{p}+\frac{q-1}{q}\right) .
$$

Thus, we have

$$
B_{m}(z, w)=\frac{m \alpha^{2 / m}}{2 \pi} \sum_{r=0}^{p-1} \frac{\zeta^{r}}{\Gamma(q(r+1) / p)}{ }_{l} F_{q}\left(1 ; \frac{\mathbf{r}+1}{\mathbf{p}}+\frac{\overrightarrow{\mathbf{j}}}{\mathbf{q}} ; \frac{\zeta^{p}}{q^{q}}\right) .
$$

Proof. By Lemma 12, we have

$G_{m}(\zeta)=\sum_{r=0}^{p-1} \frac{\zeta^{r}}{\Gamma(q(r+1) / p)} \sum_{\ell=0}^{\infty} \frac{1}{\prod_{j=0}^{q-1}((r+1) / p+(j / q))_{\ell}}\left(\frac{\zeta^{p}}{q^{q}}\right)^{\ell}$

By the definition (4), we see that

$$
{ }_{1} F_{q}\left(1 ; b_{1}, \cdots, b_{q} ; x\right)=\sum_{\ell=0}^{\infty} \frac{x^{\ell}}{\left(b_{1}\right)_{\ell} \cdots\left(b_{q}\right)_{\ell}} .
$$

It follows that

$$
\begin{aligned}
G_{m}(\zeta)= & \sum_{r=0}^{p-1} \frac{\zeta^{r}}{\Gamma(q(r+1) / p)}{ }_{1} F_{q} \\
& \cdot\left(1 ; \frac{r+1}{p}, \frac{r+1}{p}+\frac{1}{q}, \cdots, \frac{r+1}{p}+\frac{q-1}{q} ; \frac{\zeta^{p}}{q^{q}}\right) .
\end{aligned}
$$

If we use (24), then it completes the proof.

4.2. Proof of Theorem 2 (ii): Odd Numerator. Let $m=2 p+$ $1 / q$, where $2 p+1$ and $q$ are relatively prime. Then,

$$
\begin{aligned}
G_{m}(\zeta) & =\sum_{k=0}^{\infty} \frac{\zeta^{k}}{\Gamma((2 k+2) q /(2 p+1))} \\
& =\sum_{r=0}^{2 p} \zeta^{r} \sum_{\ell=0}^{\infty} \frac{\left(\zeta^{2 p+1}\right)^{\ell}}{\Gamma(2 q \ell+(2 q(r+1) /(2 p+1)))}
\end{aligned}
$$

where $k=(2 p+1) \ell+r$ with $0 \leq r \leq 2 p$. By Lemma 12 , we have

$$
\Gamma\left(2 q \ell+\frac{2 q(r+1)}{2 p+1}\right)=\Gamma(2 q t)(2 q)^{2 q \ell} \prod_{j=0}^{2 q-1}\left(t+\frac{j}{2 q}\right)_{\ell},
$$

where $t:=(r+1) /(2 p+1)$. It follows that

$$
\begin{aligned}
G_{m}(\zeta) & =\sum_{r=0}^{2 p} \frac{\zeta^{r}}{\Gamma(2 q t)} \sum_{\ell=0}^{\infty} \frac{1}{\prod_{j=0}^{2 q-1}(t+(j / 2 q))_{\ell}}\left(\frac{\zeta^{2 p+1}}{(2 q)^{2 q}}\right)^{\ell} \\
& =\sum_{r=0}^{2 p} \frac{\zeta^{r}}{\Gamma(2 q t)}{ }_{1} F_{2 q}\left(1 ; t, t+\frac{1}{2 q}, \cdots, t+\frac{2 q-1}{2 q} ; \frac{\zeta^{2 p+1}}{(2 q)^{2 q}}\right) .
\end{aligned}
$$

If we use (24), then we obtain the following.

Theorem 14 (Theorem 2 (ii) again). Let $m=(2 p+1) / q$, where $2 p+1$ and $q$ are relatively prime. Then,

$$
G_{m}(\zeta)=\sum_{r=0}^{2 p} \frac{\zeta^{r}}{\Gamma(2 q(r+1) /(2 p+1))}{ }_{1} F_{2 q}\left(1 ; \frac{\mathbf{r}+1}{2 \mathbf{p}+1}+\frac{\overrightarrow{\mathbf{j}}}{2 \mathbf{q}} ; \frac{\zeta^{2 p+1}}{(2 q)^{2 q}}\right)
$$

where

$$
\frac{\mathbf{r}+1}{2 \mathbf{p}+1}+\frac{\overrightarrow{\mathbf{j}}}{2 \mathbf{q}}=\left(\frac{r+1}{2 p+1}, \frac{r+1}{2 p+1}+\frac{1}{2 q}, \cdots, \frac{r+1}{2 p+1}+\frac{2 q-1}{2 q}\right) .
$$


Thus, we have

$B_{m}(z, w)=\frac{m \alpha^{2 / m}}{2 \pi} \sum_{r=0}^{2 p} \frac{\zeta^{r}}{\Gamma(2 q(r+1) /(2 p+1))}{ }_{1} F_{2 q}\left(1 ; \frac{\mathbf{r}+1}{2 \mathbf{p}+1}+\frac{\overrightarrow{\mathbf{j}}}{2 \boldsymbol{q}} ; \frac{\zeta^{2 p+1}}{(2 q)^{2 q}}\right)$.

\section{Special Cases}

In the last section, we express $B_{m}(z, w)$ in terms of the generalized hypergeometric series ${ }_{1} F_{q}$ for a suitable $q$. However, in general, it is difficult to find the closed form of ${ }_{1} F_{q}(1$; $\left.b_{1}, \cdots, b_{q} ; x\right)$ for any $b_{1}, \cdots, b_{q}$.

5.1. Proof of Theorem 3: The Case When $m=4$. In this case, we show that ${ }_{1} F_{1}(1,1 / 2 ; x)$ is represented in terms of the error function. In fact, we will conclude that

$$
B_{4}(z, w)=\frac{2 \alpha}{\pi} z \bar{w} e^{\alpha(z \bar{w})^{2}}(\operatorname{erf}(\sqrt{\alpha} z \bar{w})+1)+\frac{2 \sqrt{\alpha}}{\pi \sqrt{\pi}}
$$

By Proposition 9 , we need to study $\Phi(1 ; b ; x)$ for any rational number $b$ with $0<b \leq 1$. It is easy to see that $\Phi(1$ $; 1 ; x)=e^{x}$. Also, if $0<b<1$, then there is a connection between $\Phi(1 ; b ; x)$ and the incomplete gamma function.

Proposition 15. $\Phi$ satisfies the following identities.

(i) Kummer's transformation: $\Phi(a ; b ; x)=e^{x} \Phi(b-a$; $b ;-x)$

(ii) If $\mathfrak{R}(b)>\mathfrak{R}(a)$, then the confluent hypergeometric series $\Phi(a ; b ; x)$ has the integral representation

$$
\Phi(a ; b ; x)=\frac{\Gamma(b)}{\Gamma(a) \Gamma(b-a)} \int_{0}^{1} e^{x u} u^{a-1}(1-u)^{b-a-1} d u
$$

The upper incomplete gamma function $\Gamma(s, x)$ and the lower incomplete gamma function $\gamma(s, x)$ are defined by

$$
\begin{aligned}
& \Gamma(s, x)=\int_{x}^{\infty} t^{s-1} e^{-t} d t \\
& \gamma(s, x)=\int_{0}^{x} t^{s-1} e^{-t} d t
\end{aligned}
$$

Now, $\Phi(1 ; b ; x)$ can be written in terms of the lower incomplete gamma function.

Proposition 16. For any $0<b<1$, we have

$$
\Phi(1 ; b ; x)=(b-1) e^{x} x^{1-b} \gamma(b-1, x)
$$

Proof. By Proposition 15 (i), we have

$$
\Phi(1 ; b ; x)=e^{x} \Phi(b-1 ; b ;-x) .
$$

By Proposition 15 (ii), we have

$$
\begin{aligned}
\Phi(b-1 ; b ;-x) & =\frac{\Gamma(b)}{\Gamma(b-1) \Gamma(1)} \int_{0}^{1} e^{-x u} u^{b-2} d u \\
& =(b-1) x^{1-b} \int_{0}^{x} e^{-t} t^{b-2} d t=(b-1) x^{1-b} \gamma(b-1, x)
\end{aligned}
$$

It completes the proof.

In particular, if $m=4$, then we can write $G_{4}(\zeta)$ and $B_{4}($ $z, w)$ in a simple form using the error function. Recall that the error function erf $(x)$ is denoted by

$$
\operatorname{erf}(x)=\frac{2}{\sqrt{\pi}} \int_{0}^{x} e^{-t^{2}} d t
$$

It is easy to see that $\gamma(1 / 2, x)=\sqrt{\pi} \operatorname{erf}(\sqrt{x})$.

The following lemma can be proven easily by the integration parts of the integral.

Lemma 17. For any s, we have

$$
\gamma(s+1, x)=s \gamma(s, x)-x^{s} e^{-x} .
$$

By Lemma 17, we have

$$
\gamma\left(-\frac{1}{2}, x\right)=-2 \gamma\left(\frac{1}{2}, x\right)-\frac{2 e^{-x}}{\sqrt{x}}=-2 \sqrt{\pi} \operatorname{erf}(\sqrt{x})-\frac{2 e^{-x}}{\sqrt{x}} .
$$

By Proposition 16, we have

$$
\Phi\left(1, \frac{1}{2} ; x\right)=-\frac{1}{2} e^{x} \sqrt{x} \gamma\left(-\frac{1}{2}, x\right)=\sqrt{\pi x} e^{x} \operatorname{erf}(\sqrt{x})+1
$$

Now, we are ready to express $G_{4}(\zeta)$ and $B_{4}(z, w)$ in terms of the error function.

Theorem 18 (Theorem 3 again). If $m=4$, then

$$
G_{4}(\zeta)=\zeta e^{\zeta^{2}}(\operatorname{erf}(\zeta)+1)+\frac{1}{\sqrt{\pi}}
$$

Thus, we have

$$
B_{4}(z, w)=\frac{2 \alpha}{\pi} z \bar{w} e^{\alpha(z \bar{w})^{2}}(\operatorname{erf}(\sqrt{\alpha} z \bar{w})+1)+\frac{2 \sqrt{\alpha}}{\pi \sqrt{\pi}} .
$$

Proof. By Proposition 9, we have

$$
G_{4}(\zeta)=\frac{1}{\sqrt{\pi}} \Phi\left(1, \frac{1}{2} ; \zeta^{2}\right)+\zeta \Phi\left(1,1 ; \zeta^{2}\right)
$$

If we use (64) and the identity $\Phi(1,1 ; \zeta)=e^{\zeta}$, then we 
obtain (65). Since $B_{4}(z, w)=(2 \sqrt{\alpha} / \pi) G_{4}(\sqrt{\alpha} z \bar{w})$, we obtain the formula of $B_{4}(z, w)$.

5.2. Proof of Theorem 4: The Case when $m=1,2 / 3,1 / 2$. It is surprising that we can obtain the explicit forms of $B_{1}(z, w)$ , $B_{2 / 3}(z, w)$, and $B_{1 / 2}(z, w)$.

Theorem 19 (Theorem 4 (i) again). If $m=1$, then

$$
G_{1}(\zeta)=\frac{\sinh (\sqrt{\zeta})}{\sqrt{\zeta}}
$$

Thus, we have

$$
B_{1}(z, w)=\frac{\alpha}{2 \pi} \frac{\sinh \left(\alpha(z \bar{w})^{1 / 2}\right)}{(z \bar{w})^{1 / 2}}
$$

Proof. Note that $G_{1}(\zeta)={ }_{1} F_{2}(1 ; 1,3 / 2 ; \zeta / 4)$. Use the identity

$$
{ }_{1} F_{2}\left(1 ; 1, \frac{3}{2} ; x\right)=\frac{\sinh (2 \sqrt{x})}{2 \sqrt{x}}
$$
that

In fact, the identity (70) can be proven as follows. Note

$$
\begin{gathered}
{ }_{1} F_{2}\left(1 ; 1, \frac{3}{2} ; x\right)=\sum_{k=0}^{\infty} \frac{x^{k}}{(3 / 2)_{k} k !}, \\
\left(\frac{3}{2}\right)_{k} k !=\frac{3}{2} \cdot \frac{5}{2} \cdots\left(k+\frac{1}{2}\right) k !=\frac{3 \cdot 5 \cdots(2 k+1)}{2^{k}} k !=\frac{(2 k+1) !}{4^{k}} .
\end{gathered}
$$

It follows that

$$
{ }_{1} F_{2}\left(1 ; 1, \frac{3}{2} ; x\right)=\sum_{k=0}^{\infty} \frac{4^{k}}{(2 k+1) !} x^{k}=\frac{\sinh (2 \sqrt{x})}{2 \sqrt{x}} .
$$

In general, the explicit forms of the most hypergeometric series are unknown. But the very special following the hypergeometric series including (70) can be computed.

Proposition 20. For any $x$, we have

(i) ${ }_{1} F_{3}(1 ; 1,4 / 3,5 / 3 ; x)=2 e^{(-3 / 2) x^{1 / 3}} / 27 x^{2 / 3}\left\{e^{(9 / 2) x^{1 / 3}}-2\right.$ $\left.\sin \left((3 \sqrt{3} / 2) x^{1 / 3}+\pi / 6\right)\right\}$

(ii) ${ }_{1} F_{4}(1 ; 1,5 / 4,6 / 4,7 / 4 ; x)=3 / 64 x^{3 / 4}\left\{\sinh \left(4 x^{1 / 4}\right)-\right.$ $\left.\sin \left(4 x^{1 / 4}\right)\right\}$
One can find the closed forms of various hypergeometric series in [11]. In particular, one can find the closed forms of

$$
\begin{gathered}
{ }_{1} F_{3}\left(1 ; 1, \frac{4}{3}, \frac{5}{3} ; x\right)={ }_{0} F_{2}\left(; \frac{4}{3}, \frac{5}{3} ; x\right), \\
{ }_{1} F_{4}\left(1 ; 1, \frac{5}{4}, \frac{6}{4}, \frac{7}{4} ; x\right)={ }_{0} F_{3}\left(; \frac{5}{4}, \frac{6}{4}, \frac{7}{4} ; x\right),
\end{gathered}
$$

in $[12,13]$, respectively.

Now, we prove Theorem 4 (ii) and (iii) as finding the closed forms of $B_{2 / 3}(z, w)$ and $B_{1 / 2}(z, w)$ using Proposition 20. Since we have

$$
G_{2 / 3}(\zeta)=\frac{1}{2}{ }_{1} F_{3}\left(1 ; 1, \frac{4}{3}, \frac{5}{3} ; \frac{\zeta}{27}\right)
$$

it follows that

$$
B_{2 / 3}(z, w)=\frac{\alpha^{3}}{3 \pi} G_{2 / 3}\left(\alpha^{3} z \bar{w}\right)=\frac{\alpha^{3}}{6 \pi}{ }_{1} F_{3}\left(1 ; 1, \frac{4}{3}, \frac{5}{3} ; \frac{\alpha^{3} z \bar{w}}{27}\right) .
$$

By Proposition 20 (i), we have

$$
B_{2 / 3}(z, w)=\frac{\alpha e^{(-\alpha / 2)(z \bar{w})^{1 / 3}}}{9 \pi(z \bar{w})^{2 / 3}}\left\{e^{\frac{3}{2} \alpha(z \bar{w})^{1 / 3}}-2 \sin \left(\frac{\sqrt{3}}{2} \alpha(z \bar{w})^{1 / 3}+\frac{\pi}{6}\right)\right\} .
$$

Since we have

$$
G_{1 / 2}(\zeta)=\frac{1}{6}{ }_{1} F_{4}\left(1 ; 1, \frac{5}{4}, \frac{6}{4}, \frac{7}{4} ; \frac{\zeta}{4^{4}}\right)
$$

it follows that

$B_{1 / 2}(z, w)=\frac{\alpha^{4}}{4 \pi} G_{1 / 2}\left(\alpha^{4} z \bar{w}\right)=\frac{\alpha^{4}}{24 \pi}{ }_{1} F_{4}\left(1 ; 1, \frac{5}{4}, \frac{6}{4}, \frac{7}{4} ; \frac{\alpha^{4} z \bar{w}}{4^{4}}\right)$.

By Proposition 20 (ii), we have

$$
B_{1 / 2}(z, w)=\frac{\alpha}{8 \pi(z \bar{w})^{3 / 4}}\left\{\sinh \left(\alpha(z \bar{w})^{1 / 4}\right)-\sin \left(\alpha(z \bar{w})^{1 / 4}\right)\right\}
$$

It completes the proof of Theorem 4 (ii) and (iii).

5.3. Proof of Theorem 5. In this section, $A(x) \sim B(x)$ means that $A(x) / B(x)$ converges to a nonzero constant as $x$ goes to some number or infinity.

Theorem 21 (Theorem 5 again). Let $m$ be any positive even integer. Then,

$$
B_{m}(z, z) \sim e^{\alpha|z|^{m}}|z|^{m-2} a s|z| \longrightarrow \infty .
$$


Proof. Let $m=2 p$. Then, by Theorem 1 (i),

$$
\begin{aligned}
B_{2 p}(z, w) & =\frac{p \alpha^{1 / p}}{2 \pi} \sum_{r=0}^{p-1} \frac{\zeta^{r}}{\Gamma((r+1) / p)} \Phi\left(1 ; \frac{r+1}{p} ; \zeta^{p}\right) \\
& =\frac{p \alpha^{1 / p}}{2 \pi}\left\{\sum_{r=0}^{p-2} \frac{\zeta^{r}}{\Gamma((r+1) / p)} \Phi\left(1 ; \frac{r+1}{p} ; \zeta^{p}\right)+\zeta^{p-1} \Phi\left(1 ; 1 ; \zeta^{p}\right)\right\}
\end{aligned}
$$

If $0 \leq r \leq p-2$, then by Proposition 16,

$$
\Phi\left(1 ; \frac{r+1}{p} ; \zeta^{p}\right)=\left(\frac{r+1}{p}-1\right) e^{\zeta^{p}} \zeta^{p-r-1} \gamma\left(\frac{r+1}{p}-1, \zeta^{p}\right)
$$

and $\Phi\left(1 ; 1 ; \zeta^{p}\right)=e^{\zeta^{p}}$. It follows that

$$
B_{2 p}(z, w)=\frac{p \alpha^{1 / p}}{2 \pi}\left\{\sum_{r=0}^{p-2} e^{\zeta^{p}} \zeta^{p-1} \frac{\gamma\left(((r+1) / p)-1, \zeta^{p}\right)}{\Gamma((r+1) / p-1)}+\zeta^{p-1} e^{\zeta^{p}}\right\} .
$$

Since $\gamma((r+1) / p-1, x) \longrightarrow \Gamma((r+1) / p)$ as $x \longrightarrow \infty$, it completes the proof.

In fact, it is easily checked that (80) holds also when $m$ $=1,3 / 2,1 / 2$ using the explicit forms in Theorem 4 . We can conjecture that (80) holds for any $m>0$.

\section{Concluding Remarks}

In fact, we can consider the more generalized Fock space. Let $d \lambda_{\phi}(z)=c_{\phi} e^{-\phi(z)} d A(z)$, where $d A(z)$ is the Euclidean area measure on the complex plane $\mathbb{C}$. We assume that $\phi(r)$ is radial and increasing on $[0, \infty)$ with $\lim _{r \rightarrow \infty} \phi(r)=\infty$. We call the (generalized) Fock space $F_{\phi}^{2}(\mathbb{C})$ as the set of all entire functions $f$ in $L^{2}\left(\mathbb{C}, d \lambda_{\phi}\right)$. Another simple example is $\phi(r)=\ln r$. In this case, we can show that the Fock kernel can be written in terms of the Meijer-G function. It will be interesting that one finds the relation between the other hypergeometric series and the new Fock kernel with respect to $\phi$.

\section{Data Availability}

No data were used to support this study.

\section{Conflicts of Interest}

The author declares that he has no conflicts of interest.

\section{Acknowledgments}

This work was supported by NRF-2018R1D1A1B07050044 from National Research Foundation of Korea.

\section{References}

[1] K. Zhu, Analysis on Fock Spaces, Graduate Texts in Mathematics, Springer, New York, NY, USA, 2012.

[2] H. R. Cho, J.-D. Park, and K. Zhu, "Products of Toeplitz operators on the Fock space," Proceedings of the American Mathematical Society, vol. 142, no. 7, pp. 2483-2489, 2014.

[3] T. Beberok, "Explicit formulas of the Bergman kernel for some Reinhardt domains," Journal of Geometric Analysis, vol. 26, no. 4, pp. 2883-2892, 2016.

[4] G. Francsics and N. Hanges, "The Bergman kernel of complex ovals and multivariable hypergeometric functions," Journal of Functional Analysis, vol. 142, no. 2, pp. 494-510, 1996.

[5] T. Beberok, "The Bergman kernel for intersection of two complex ellipsoids," Bulletin of the Korean Mathematical Society, vol. 53, no. 5, pp. 1291-1308, 2016.

[6] J.-D. Park, "New formulas of the Bergman kernels for complex ellipsoids in $\mathbb{C}^{2}$, " roceedings of the American Mathematical Society, vol. 136, no. 12, pp. 4211-4221, 2008.

[7] J.-D. Park, "Explicit formulas of the Bergman kernel for 3dimensional complex ellipsoids," Journal of Mathematical Analysis and Applications, vol. 400, no. 2, pp. 664-674, 2013.

[8] J. Gonessa, "Duality of Fock spaces with respect to the minimal norm," Archiv der Mathematik, vol. 100, no. 5, pp. 439-447, 2013.

[9] C. Cascante, J. Fàbrega, and D. Pascuas, "Boundedness of the Bergman projection on generalized Fock-Sobolev spaces on $\mathbb{C}^{\mathrm{n}}$, " Complex Analysis and Operator Theory, vol. 14, no. 2, 2020.

[10] H. R. Cho, H. Choi, and H.-W. Lee, "Explicit formula for the reproducing kernels for some weighted Fock spaces," Journal of Mathematical Analysis and Applications, vol. 497, no. 2, article 124920, 2021.

[11] https://functions.wolfram.com/HypergeometricFunctions/ HypergeometricPFQ/03/01/.

[12] https://functions.wolfram.com/HypergeometricFunctions/ HypergeometricPFQ/03/01/15/.

[13] https://functions.wolfram.com/HypergeometricFunctions/ HypergeometricPFQ/03/01/19/. 